\title{
Unilateral bronchospasm during one-lung ventilation
}

\author{
Taisuke Yokota $\cdot$ Takashi Asai $\cdot$ Yasuhisa Okuda
}

Received: 17 November 2014 / Accepted: 22 December 2014 / Published online: 24 January 2015

(C) Japanese Society of Anesthesiologists 2015

\section{To the Editor:}

We experienced an unreported case of the absence of end-tidal carbon dioxide concentration $\left(\mathrm{EtCO}_{2}\right)$ waveforms during one-lung ventilation: unilateral bronchospasm [1]. A 76-year-old man with left lung cancer, mild emphysema, and possible asthma cough was scheduled for video-assisted thoracic surgery. After induction of general anesthesia, a 37 Fr. left-sided double-lumen tube (DLT) was inserted, and one-lung ventilation was initiated. Several minutes later, the $\mathrm{EtCO}_{2}$ waveforms suddenly became completely flat. Manual ventilation of the right dependent lung was attempted but was impossible. Immediate fiberoptic bronchoscopy did not detect malposition of the DLT or an obstruction of the patient's airway. The anesthesia machine was confirmed to be functioning normally, and there was no obstruction of a gas sampling tube. Auscultation of the right chest showed no gas entry. A chest X-ray taken several minutes later with the patient in the supine position ruled out pneumothorax but showed decreased air entry to the right lung, indicating unilateral bronchospasm. With infusion of aminophylline $250 \mathrm{mg}$, it became possible to ventilate the right lung, and the $\mathrm{EtCO}_{2}$ waveforms reappeared. Auscultation of the chest showed weak wheezing. The causes of impossible ventilation during one-lung ventilation are various (see Table 1 in supplementary material). When ventilation becomes impossible during one-lung ventilation in the absence of the $\mathrm{EtCO}_{2}$, we should consider that unilateral bronchospasm is one possible cause.

\section{Reference}

1. Asai T. Monitoring during difficult airway management. J Anesth. 2014;28:87-93.

Electronic supplementary material The online version of this article (doi:10.1007/s00540-014-1970-z) contains supplementary material, which is available to authorized users.

T. Yokota $(\bowtie) \cdot$ T. Asai $\cdot$ Y. Okuda

Department of Anesthesiology, Koshigaya Hospital, Dokkyo

Medical University, 2-1-50 Minamikoshigaya, Koshigaya City,

Saitama 343-8555, Japan

e-mail: intensive2345@yahoo.co.jp 